DIGITALCOMMONS - @WAYNESTATE -
Clinical Research in Practice: The Journal of Team Hippocrates

Volume 4 | Issue 1

Article 4

2018

\title{
Closure of patent foramen ovale or medical therapy alone are both appropriate choices for preventing recurrent cryptogenic strokes
}

Kevin Juan Zhang

Wayne State University School of Medicine, kezhang@med.wayne.edu

Follow this and additional works at: https://digitalcommons.wayne.edu/crp

Part of the Medical Education Commons, and the Translational Medical Research Commons

\section{Recommended Citation}

ZHANG KJ. Closure of patent foramen ovale or medical therapy alone are both appropriate choices for preventing recurrent strokes after cryptogenic stroke. Clin. Res. Prac. 2018;4(1):eP1474. doi: 10.22237/crp/1518739380

This Critical Analysis is brought to you for free and open access by the Open Access Journals at DigitalCommons@WayneState. It has been accepted for inclusion in Clinical Research in Practice: The Journal of Team Hippocrates by an authorized editor of DigitalCommons@WayneState. 


\title{
Closure of patent foramen ovale or medical therapy alone are both appropriate choices for preventing recurrent cryptogenic strokes
}

KEVIN JUAN ZHANG, Wayne State University School of Medicine, kezhang@med.wayne.edu

\begin{abstract}
A critical appraisal and clinical application of Carroll JD, Saver JL, Thaler DE, et al. Closure of patent foramen ovale versus medical therapy after cryptogenic stroke. N Engl J Med, 2013;368(12):1092-1100. doi: 10.1056/nejmoa1301440.
\end{abstract}

Keywords: $\quad$ Patent foramen ovale, closure, anticoagulation, medical therapy, cryptogenic stroke

\section{Clinical Context}

A 34-year-old woman with a past medical history of migraine headaches with auras, a stroke in 2016, and a patent foramen ovale (PFO) presented to the emergency department with a 9-day history of migraine headaches and dizziness. She described her migraines as left-sided pulsating headaches with photophobia and noise sensitivity. She uses acetaminophen, butalbital, and caffeine or ibuprofen in an acute setting. She was on topiramate $75 \mathrm{mg}$ for migraine prophylaxis. She noted that she has had increasing episodes of migraine headaches since her stroke 8 months ago. At that time, she received a thorough workup to rule out common causes of stroke. The workup returned negative, so it was deemed to be a cryptogenic stroke. Upon arrival in the emergency department (ED), her headaches and dizziness had begun to resolve. Her neurologist recommended increasing the dosage of her topiramate from $75 \mathrm{mg}$ BID to $100 \mathrm{mg}$ BID. Her medication list since her stroke also included aspirin $81 \mathrm{mg}$, with which she was non-adherent. We stressed the importance of adherence to her medication and added a statin to her medications. She asked, "Should I have my PFO closed?"

\section{Clinical Question}

Is there a benefit in preventing recurrent strokes with PFO closure over anticoagulation alone for a patient who has had a cryptogenic stroke?

\section{Research Article}

Carroll JD, Saver JL, Thaler DE, et al. Closure of patent foramen ovale versus medical therapy after cryptogenic stroke. $N$ Engl J Med, 2013;368(12):1092-1100. doi: 10.1056/nejmoa1301440.

KEVIN JUAN ZHANG is a student at Wayne State University School of Medicine. 
ZHANG KJ. Closure of patent foramen ovale or medical therapy alone are both appropriate choices for preventing recurrent strokes after cryptogenic stroke. Clin. Res. Prac. 2018;4(1):eP1474. doi: $10.22237 / \mathrm{crp} / 1518739380$
Clinical Research in Practice

The Journal of Team Hippocrates

VOL 4 ISS 1 / eP1474 / FEBRUARY 16, 2018 doi: $10.22237 / \mathrm{crp} / 1518739380$

\section{Related Literature}

Patients with PFOs possess an inter-atrial shunt that allows emboli originating from the venous system to traverse directly into systemic circulation, causing a paradoxical embolism. 1 The current management of patients who have a PFO and have had a cryptogenic stroke is either anticoagulation with aspirin, clopidogrel, or aspirin with dipyridamole, or closure of the PFO followed by 6 months of anticoagulation.

A literature review was conducted in PubMed. Keywords included "closure of patent foramen ovale," "medical therapy," "cryptogenic stroke," and "clinical trial." Several articles were applicable to the clinical question.

A Cochrane Review by Li et al. demonstrated that there was no significant difference in recurrence of strokes between patients receiving PFO closure as opposed to medical therapy alone. $\underline{2}$ Without knowing the inclusion criteria for these trials, it is unknown whether our patient fits the population studied. Without authentication of homogeneity of these RCTs, the validity of the results from combining these RCTs is uncertain. The authors considered this study at high risk for bias. An article by Kent et al. was

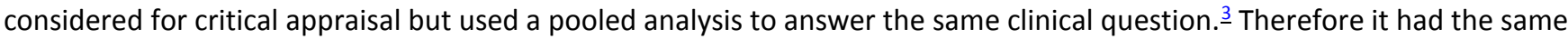
limitations and methodological faults that the pooled studies possessed. It was determined that the study by Caroll et al. demonstrated a thoughtful protocol and analysis, being an RCT with matching baseline characteristics between the two groups. Our patient fits the inclusion criteria for the study.

\section{Critical Appraisal}

Carroll et al. conducted a prospective, multicenter, controlled, and randomized clinical trial with blinded adjudication of end-point events testing whether there was a benefit for PFO closure over medical therapy alone for preventing stroke recurrence for patients who have had a cryptogenic stroke. This article is considered a level $1 \mathrm{~b}$ study, as an individual RCT with a narrow confidence interval, according to the Oxford Centre for Evidence-based Medicine. Inclusion criteria involved patients between 18 and 60 years of age who had a cryptogenic stroke within the past 270 days and had a PFO identified by transesophageal echocardiography (TEE), which closely fits our own patient. In addition, patients must have demonstrated evidence of new cerebral infarct on MRI or evidence of focal neurological deficits lasting more than 24 hours.

In the intention-to-treat analysis, there were 0.66 nonfatal ischemic strokes per 100 patient years in the closure group compared to 1.38 nonfatal ischemic strokes per 100 patient years in the medical-therapy group (Hazard Ratio 0.49, $p=0.08$ ). There was superiority in PFO closure in the per-protocol analysis with $p$-values of 0.03 and 0.007 , respectively. These values differed from the intention-to-treat analysis, since it considered patients according to treatments they ultimately chose.

A notable strength demonstrated in this study is that it maintains its integrity by ultimately reporting the results from the intentionto-treat analysis as its conclusive finding. The closure and medical-therapy groups were similar on baseline characteristics, which aids the interpretation of the results. The per-protocol analysis is not as resistant to bias since patients who did not complete their assignment, and therefore dropped out of this analysis, may have differed in significant ways. The as-treated analysis compared patients according to the treatment they received; therefore they may have changed from their original assignment due to a significant reason. This form of analysis is essentially introducing selection bias. Using sub-group analysis, the researchers concluded that the per-protocol and as-treated analyses showed that closure of PFO was favored in patients with an atrial septal aneurysm and a substantial shunt size. These types of conclusions are useful only for hypothesis generation.

There are several limitations to this study. Patients were excluded if they did not have a true cryptogenic stroke and had an identifiable cause for their paradoxical emboli. This is only possible if each of these patients received a full and proper workup, including proper interpretation of imaging and hypercoagulable workup. In addition, the mean follow-up time was only 2.6 years. For those receiving medical therapy alone, it's recommended that these patients receive anticoagulation for the rest of their lives. Therefore, the amount of time from their cryptogenic stroke in addition to the mean follow-up time is small in comparison to the total amount of time they would receive anticoagulation. 
ZHANG KJ. Closure of patent foramen ovale or medical therapy alone are both appropriate choices for preventing recurrent strokes after cryptogenic stroke. Clin. Res. Prac. 2018;4(1):eP1474. doi: $10.22237 / \mathrm{crp} / 1518739380$
Clinical Research in Practice

The Journal of Team Hippocrates

VOL 4 ISS 1 / eP1474 / FEBRUARY 16, 2018 doi: $\underline{10.22237 / \mathrm{crp} / 1518739380}$

\section{Clinical Application}

Patients with PFOs have an inter-atrial shunt, putting them at risk for paradoxical emboli originating from venous circulation that can lead to strokes rather than pulmonary emboli. In patients with PFOs in whom a paradoxical or cryptogenic stroke is suspected, a careful workup must be done to rule out causative factors such as a hypercoagulable state, prothrombin mutation, or atrial fibrillation, for which patients with PFO are at a higher rate of vulnerability. 4

Patients with PFO need to either be on lifelong anticoagulation or have PFO closure in order to reduce their risk of cryptogenic stroke. It has been demonstrated that these patients can choose either form of management with similar protection from recurrent strokes, in the absence of some extra risk factors that were not present in our patient. When we discussed these options with our patient, she chose lifelong anti-coagulation. We counseled her on the importance of taking her medication every day and the risks of missing doses with her PFO. She indicated understanding, and we were comfortable that she would have better anti-coagulation compliance moving forward. She has been compliant on a regimen of aspirin $81 \mathrm{mg}$ and remains stroke-free at last known contact (at least six months).

Take-home points:

1. Patients with PFOs who have had a cryptogenic stroke can be managed with either PFO closure or medical therapy alone. Those with an atrial septal aneurysm or a substantial shunt size may benefit from PFO closure compared to medical therapy alone.

2. While anti-coagulation is as effective as closure for reducing stroke risk in PFO patients with good medication compliance, poor compliance can reduce the effectiveness. Patients need to be appropriately counseled on this risk when choosing a treatment.

\section{References}

1. Di Tullio M, Sacco RL, Gopal A, Mohr JP, Homma S. Patent foramen ovale as a risk factor for cryptogenic stroke. Ann Intern Med. 1992;117(6):461-465. doi: 10.7326/0003-4819-117-6-461

2. Li J, Liu J, Liu M, et al. Closure versus medical therapy for preventing recurrent stroke in patients with patent foramen ovale and a history of cryptogenic stroke or transient ischemic attack. Cochrane Database Syst Rev. 2015(9):CD009938. doi: 10.1002/14651858.CD009938.pub2

3. Kent DM, Dahabreh IJ, Ruthazer R, et al. Device closure of patent foramen ovale after stroke: pooled analysis of completed randomized trials. J Am Coll Cardiol. 2016;67(8):907-917. doi: 10.1016/j.jacc.2015.12.023

4. Berthet K, Lavergne T, Cohen A, et al. Significant association of atrial vulnerability with atrial septal abnormalities in young patients with ischemic stroke of unknown cause. Stroke. 2000;31(2):398-403. doi: 10.1161/01.STR.31.2.398 